\title{
HOMOGENIZATION OF A CAPILLARY PHENOMENA
}

\author{
N. Labani And M. Mabrouk
}

\begin{abstract}
We study the height of a liquid in a tube when it contains a great number of thin vertical bars and when its border is finely strained. For this, one uses an epi-convergence method.
\end{abstract}

\section{Introduction}

Let $T$ be a cylindrical tube of height infinity, containing a compressible liquid and a great number of thin cyclindrical bars of radius $r$ which are distributed periodically with a period $\epsilon$ such that $r<\epsilon$ (see Figure 1). One supposes the bars are of the same material which is specified by the coefficient $\lambda$ (see (1) below). In section 2 we show that (see Proposition 2 and Theorem 1$)$ when $(\epsilon, r \lambda)$ tends to $(0,0,0)$ the height of the liquid grows indefinitely, does not grow or take a certain height according to the rate $\frac{r \lambda}{\epsilon^{2}}$ being $+\infty, 0$ or finite (see Proposition 2 and Theorem 1).

Suppose now that the border of the tube is made of two alternate periodic bands with a period $\epsilon$. The band 1 of length $r$ is encrusted in the other of length $\epsilon-r(r<\epsilon)$ (see Figure 3). The different materials that constitute the bands are specified by the coefficients $k_{1}$ and $k_{2}$.

Keywords. Homogenization, epi-convergence, capillarity. 
One shows that (see Theorem 2) when $(\epsilon, r)$ tends to $(0,0)$ the tube behaves like an intermediate and homogeneous one of coefficient $k_{1} a+$ $k_{2}(1-a)$, where $a=\lim _{(\epsilon, r) \rightarrow(0,0)} \frac{r}{\epsilon}$.

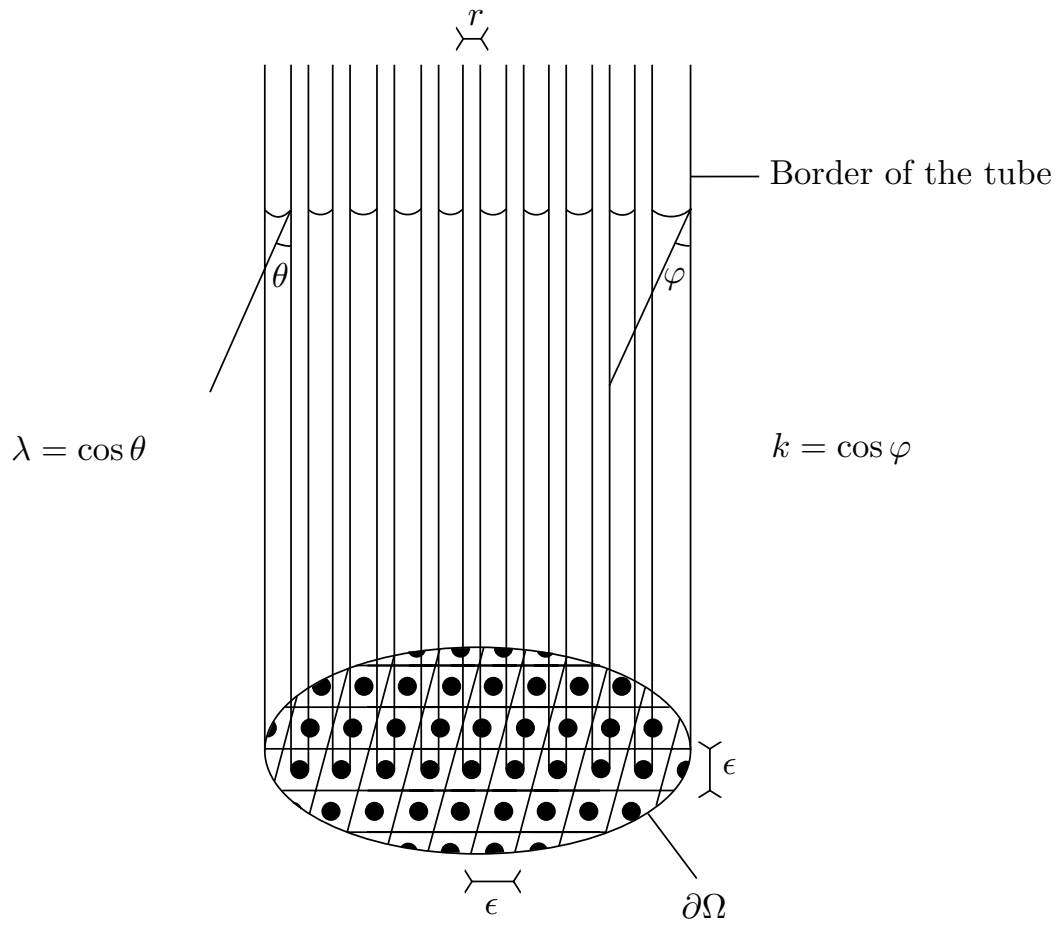

Figure 1. The tube with the bars.

\section{Tube containing a great number of thin bars}

\subsection{Problem.}

Let $T$ be a cylindrical tube of base $\Omega$ with radius $R$ and of height infinity, containing a compressible liquid of a finite volume. Let us divide $\Omega$ by a net of side $\epsilon$ and let us set in the center $x_{\epsilon, j}$ of the net $Y_{\epsilon, j}(j \in I$ the integer part of the real $\left.\frac{|\Omega|}{\epsilon^{2}}\right)$ an homogeneous vertical cylindrical bar $T_{h, j}$ of radius $r(r<\epsilon)$ where $h=(\epsilon, r, \lambda)$ (see Figures 1 and 2 ).

Let us denote the union of the bars $T_{h, j}$ by $T_{h}$ and let $\Omega_{h}=\Omega \backslash T_{h}$. 


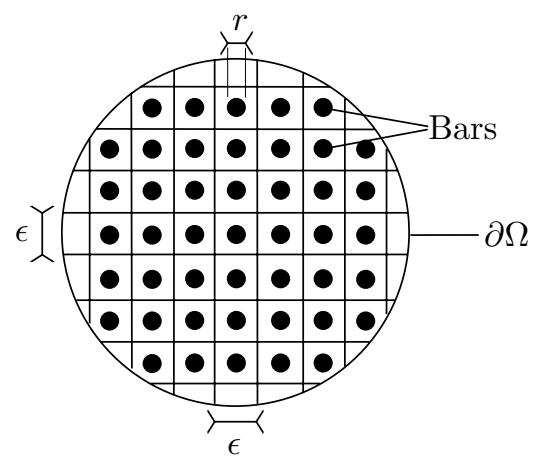

Figure 2

The height of the liquid is stabilizing when it satisfies the following minimization problem (see $[\mathbf{3}],[\mathbf{4}]$ ):

$$
\operatorname{Min}\left\{J_{h}(v), v \in B V\left(\Omega_{h}\right)\right\}
$$

where

$$
J_{h}(v)=\int_{\Omega_{h}} \sqrt{1+|D v|^{2}}+c \int_{\Omega_{h}} v^{2}-\lambda \int_{\partial T_{h}} v-k \int_{\partial \Omega} v
$$

for $v \in B V\left(\Omega_{h}\right)$ the space of functions of bounded variation defined in $\Omega$ (see [5] for the definition and the properties of $B V(\Omega)$ ):

$$
B V(\Omega)=\left\{v \in L^{1}(\Omega), \int_{\Omega}|D v|<+\infty\right\}
$$

where

$$
\int_{\Omega}|D v|=\sup \left\{\sum_{k=1}^{n} \int_{\Omega} v(x) \frac{\partial \phi_{k}}{\partial x_{k}}(x) d x, \sum_{k=1}^{n} \phi_{k}^{2}(x) \leq 1, \phi_{k} \in C_{0}^{1}(\Omega) \forall k\right\} .
$$

$C_{0}^{1}(\Omega)$ is the space of functions defined in $\Omega$, of class $C^{1}$ with a compact support included in $\Omega$,

$$
\begin{aligned}
\int_{\Omega} \sqrt{1+|D v|^{2}} & =\sup \left\{\int_{\Omega} v(x) \phi_{0}(x) d x\right. \\
- & \left.\sum_{k=1}^{n} \int_{\Omega} v(x) \frac{\partial \phi_{k}}{\partial x_{k}}(x) d x, \sum_{k=0}^{n} \phi_{k}^{2}(x) \leq 1, \phi_{k} \in C_{0}^{1}(\Omega) \forall k\right\} .
\end{aligned}
$$


The capillary constant $c$ is related to the pressure term of the liquid, $\lambda$ and $k$ are respectively the cosine of the contact angles (liquid, bar), (liquid, border) and which specify respectively the bars material and the tube border. The constants $c, \lambda$ and $k$ are supposed real positive numbers.

Problem. We look for the limit behavior of the height of the liquid when $h=(\epsilon, r, \lambda)$ tends to $(0,0,0)$.

\subsection{Existence and extension of the solution.}

Proposition 1. (See [3], [4]). The problem (1) has a unique positive solution in $B V\left(\Omega_{h}\right)$. Furthermore $u_{h} \in C^{2}\left(\overline{\Omega_{h}}\right)$ and is constant on the boundary $\partial T_{h, j}$ of the bar $T_{h, j}$ for all $j \in I$.

Let us cite a trace lemma in the space $B V(\Omega)$ which will be useful:

Lemma 1. (See [4]). Suppose that $\Omega$ is of class $C^{2}$ and satisfying an internal sphere condition of radius $r$ (i.e. for any boundary point $x \in \partial \Omega$, there is a ball $B$ of radius $r$ such that $B \subset \Omega$ and $x \in \partial B)$. Then there exists a positive constant ct such that for any $v \in B V(\Omega)$,

$$
\int_{\partial \Omega}|v| \leq \int_{\Omega}|D v|+\frac{c t}{r} \int_{\Omega}|v|
$$

Let us now state some estimates of $u_{h}$ :

Proposition 2. Let $a=\lim _{h \rightarrow 0} \frac{r \lambda}{\epsilon^{2}}$. Then,

i) If $a=+\infty$, then $\lim _{h \rightarrow 0} \int_{\Omega_{h}} u_{h}=+\infty$ and if $a$ is finite, then

$$
\lim _{h \rightarrow 0} \int_{\Omega_{h}} u_{h}=\frac{k|\partial \Omega|}{2 c}+\frac{\pi a|\Omega|}{c} .
$$

ii) If a is finite, then $\int_{\Omega_{h}} u_{h}$ and $\int_{\Omega_{h}}\left|D u_{h}\right|$ are uniformly bounded for $\frac{\lambda}{\epsilon}$ bounded.

Proof: i) The problem (1) is equivalent to the Euler equation (see [4])

$$
\begin{array}{rll}
-\operatorname{Div} T u_{h}+2 c u_{h}=0 & \text { in } & \Omega_{h} \\
T u_{h} \cdot n=\lambda & \text { on } & \partial T_{h} \\
T u_{h} \cdot n=k & \text { on } & \partial \Omega,
\end{array}
$$

where $T v=\frac{D v}{\sqrt{1+|D v|^{2}}}$ and $n$ the outer normal. 
Multiplying (2) by the characteristic function $\chi_{\Omega_{h}}$ and using the Green formula, (3) and (4),

$$
\int_{\Omega_{h}} u_{h}=\frac{k|\partial \Omega|}{2 c}+\frac{\pi|\Omega|}{c} \frac{\lambda r}{\epsilon^{2}},
$$

hence passing to the limit in (5), one obtains i).

ii) According to Lemma 1

$$
\int_{\partial T_{h}} u_{h} \leq \int_{\Omega_{h}}\left|D u_{h}\right|+\frac{c t}{\epsilon} \int_{\Omega_{h}} u_{h}
$$

Let $\tilde{u}_{h}$ be the extension of $u_{h}$ in $\Omega$ defined by

$$
\begin{aligned}
& \tilde{u}_{h}=u_{h} \quad \text { in } \quad \Omega_{h} \\
& \tilde{u}_{h}=u_{h, j} \quad \text { in } \quad T_{h, j} ; j \in I,
\end{aligned}
$$

where $u_{h, j}$ is the value of $u_{h}$ on $\partial T_{h, j}$.

Using again Lemma 1, one has

(9)

$$
\begin{aligned}
\int_{\partial h} u_{h} & =\int_{\partial h} \tilde{u}_{h} \leq \int_{\Omega}\left|D \tilde{u}_{h}\right|+\frac{c t}{R} \int_{\Omega} \tilde{u}_{h} \\
& =\int_{\Omega}\left|D u_{h}\right|+\frac{c t}{R} \int_{\Omega_{h}} u_{h}+\frac{c t}{R} \int_{T_{h}} u_{h} \\
& =\int_{\Omega}\left|D u_{h}\right|+\frac{c t}{R} \int_{\Omega_{h}} u_{h}+c t \frac{r}{R} \int_{\partial T_{h}} u_{h} \\
& \leq(1+c t . r) \int_{\Omega_{h}}\left|D u_{h}\right|+c t \int_{\Omega_{h}} u_{h}
\end{aligned}
$$

where the last inequality of (9) is due to (6).

The inequality

$$
J_{h}\left(u_{h}\right) \leq J_{h}(0)=|\Omega|,
$$

(6) and (9) yield

$$
(1-k-\lambda-k r) \int_{\Omega_{h}}\left|D u_{h}\right|+c \int_{\Omega} u_{h}^{2} \leq c t\left(1+\frac{\lambda}{\epsilon}\right) .
$$

Hence the assertion ii) holds.

The following proposition yields an extension of $u_{h}$ and of $J_{h}$ in $\Omega$ which will be useful afterwards. 
Proposition 3. Suppose that $\lim _{h \rightarrow 0} \frac{\lambda}{\epsilon}$ is finite. Let $\tilde{u}_{h}$ be the extension of $u_{h}$ in $\Omega$ which is defined by (7), (8) and $\tilde{J}_{h}$ the extension of $J_{h}$ in $B V(\Omega)$ defined by

$$
\tilde{J}_{h}(v)=\int_{\Omega} \sqrt{1+|D v|^{2}}+c \int_{\Omega_{h}} v^{2}-\lambda \int_{\partial T_{h}} v_{i}-k \int_{\partial \Omega} v,
$$

where $v_{i}$ is the trace of the restriction of $v$ in $\Omega_{h}$. Then

i) $\tilde{J}_{h}$ attains its minimum at the point $\tilde{u}_{h}$.

ii) The sequence $\tilde{u}_{h}$ is uniformly bounded in $B V(\Omega)$. In particular a subsequence of $\tilde{u}_{h}$, noted by $\tilde{u}_{h}$, converges to an element $u(u \in$ $B V(\Omega))$ in $L^{1}(\Omega)$.

Proof: i) For the existence and the regularity of the minimum of $\tilde{J}_{h}$ in $B V(\Omega)$ see $[\mathbf{3}],[4]$. The minimum value of $\tilde{J}_{h}$ is attained at $\tilde{u}_{h}$ since $\tilde{J}_{h}\left(\tilde{u}_{h}\right)=J_{h}\left(u_{h}\right)$.

ii) From the definition of $\tilde{u}_{h}$,

$$
\int_{\Omega}\left|D \tilde{u}_{h}\right|=\int_{\Omega_{h}}\left|D u_{h}\right|
$$

On the other hand, using (6), one has

$$
\int_{\Omega} \tilde{u}_{h}=\int_{\Omega_{h}} u_{h}+\frac{r}{2} \int_{\partial T_{h}} u_{h} \leq\left(1+c t \frac{r}{\epsilon}\right) \int_{\Omega_{h}} u_{h}+\frac{r}{2} \int_{\Omega_{h}}\left|D u_{h}\right| .
$$

Then, using Proposition 2, (10) and (11), one deduces that $\tilde{u}_{h}$ is uniformly bounded in $B V(\Omega)$ if $\lim _{(\lambda, \epsilon) \rightarrow(0,0)} \frac{\lambda}{\epsilon}$ is finite.

\subsection{Epi-limit of the functional $\tilde{J}_{h}$.}

The following proposition characterizes the epi-limit of the sequence $\tilde{J}_{h}$ (see $[\mathbf{1}],[\mathbf{2}]$ for the definition and the properties of the epi-limit) when $h \rightarrow(0,0,0)$ :

Proposition 4. Suppose that $a=\lim _{h \rightarrow 0} \frac{\lambda r}{\epsilon^{2}}$ is finite. Then the sequence of the functionals $\tilde{J}_{h}$ epi-converges to the functional $J$ in $B V(\Omega)$ endowed with the topology of $L^{1}(\Omega)$ where

$$
J(v)=\int_{\Omega} \sqrt{1+|D v|^{2}}+c \int_{\Omega} v^{2}-2 \pi a \int_{\Omega} v-k \int_{\partial \Omega} v
$$

for $v \in B V(\Omega)$. 
Proof: 1) Let $v \in B V(\Omega)$ and $v_{h}=v$. Since

$$
\lambda \int_{\partial T_{h}} v=\frac{r \lambda}{\epsilon^{2}} \sum_{j \in I} \epsilon^{2}\left(\frac{1}{\left|\partial T_{h, j}\right|} \int_{\partial T_{h, j}} v\right),
$$

then

$$
\lim _{h \rightarrow 0} \tilde{J}_{h}\left(v_{h}\right)=J(v)
$$

2) Let $v$ and $v_{h} \in B V(\Omega)$ such that $v_{h}$ converges to $v$ in $L^{1}(\Omega)$ when $h$ tends to $(0,0,0)$. Let $J_{h}^{\prime}$ the functional defined in $B V(\Omega)$ by

$$
J_{h}^{\prime}(v)=\int_{\Omega} \sqrt{1+|D v|^{2}}+c \int_{\Omega_{h}} v^{2}-\lambda \int_{\partial T_{h}} v_{i}-k \int_{\partial \Omega} v,
$$

where $v_{i}$ is the trace, on $\partial T_{h}$, of the restriction of $v$ to $\Omega_{h}$.

Using the inequality (6),

$$
\begin{aligned}
J_{h}^{\prime}(v)-\tilde{J}_{h}\left(v_{h}\right) \leq & \left(\int_{\Omega} \sqrt{1+|D v|^{2}}-k \int_{\partial \Omega} v\right) \\
& -\left(\int_{\Omega} \sqrt{1+\left|D v_{h}\right|^{2}}-k \int_{\partial \Omega} v_{h}\right) \\
& +\int_{\partial T_{h}}\left|v_{h}-v\right|+c\left(\int_{\Omega} v^{2}-\int_{\Omega_{h}} v_{h}^{2}\right) \\
\leq & \left(\int_{\Omega} \sqrt{1+|D v|^{2}}-k \int_{\partial \Omega} v\right) \\
& -\left((1-\lambda) \int_{\Omega} \sqrt{1+\left|D v_{h}\right|^{2}}-k \int_{\partial \Omega} v_{h}\right) \\
& +c t \frac{\lambda}{\epsilon} \int_{\Omega}\left|v_{h}-h\right|+\lambda \int_{\Omega}|D v| \\
& +c\left(\int_{\Omega} v^{2}-\int_{\Omega_{h}} v_{h}^{2}\right) .
\end{aligned}
$$

Now, passing to the limitinf in (12), using the lower semi continuity of the functionsl $v \rightarrow \int_{\Omega} \sqrt{1+|D v|^{2}}-k \int_{\partial \Omega} v$ in $B V(\Omega)$ according to the topology of $L^{1}(\Omega)$ (see $[6]$ ), Fatou Lemma, the fact that $v_{h}$ converges to $v$ in $L^{1}(\Omega)$ and that $\lim _{h \rightarrow 0} \frac{\lambda}{\epsilon}$ is finite, one deduces that

$$
J(v)=\liminf _{h \rightarrow 0} J_{h}^{\prime}(v) \leq \liminf _{h \rightarrow 0} \tilde{J}_{h}\left(v_{h}\right) .
$$




\subsection{Limit behavior.}

According to the properties of the epi-convergence (see [1], [2]), one deduces the limit behavior of the problem (1) from Propositions 2 and 3.

Theorem 1. If $\lim _{h \rightarrow 0} \frac{\lambda r}{\epsilon^{2}}$ is finite, then the minimum $\tilde{J}_{h}\left(\tilde{u}_{h}\right)$ of $\tilde{J}$ converges to the minimum $J(u)$ and $\tilde{u}_{h}$ converges to $u$ in $L^{1}(\Omega)$ when $h$ tends to $(0,0,0)$.

Physic interpretation. When the section, of radius $r$, of the bars $T_{h, j}$ satisfies $r \gg \frac{\epsilon^{2}}{\lambda}$, the liquid rises indefinitely; when $r \ll \frac{\epsilon^{2}}{\lambda}$, it does not rise and when $r \simeq a \frac{\epsilon^{2}}{\lambda}, a \in(0,+\infty)$, the capillary problem in the tube with the bars is approximated by a capillary problem in the tube without bars with the same capillary constant $2 c$ but with $-2 \pi a$ as Lagrange parameter.

\section{Tube with a strained border}

Let $n \in N^{*}$ and $\epsilon=\frac{|\Omega|}{n}$. Suppose now that the border of the tube is formed by two bands which are distributed alternately and periodically with the period $\epsilon$ (see Figures 3 and 4): the boundary $\partial \Omega$ is formed by $n \operatorname{arcs} Y_{\epsilon, j}, j \in\{1, \ldots, n\}$ of length $\epsilon$. In the center of any arc $Y_{\epsilon, j}$ is encrusted an arc $T_{h, j}$ (band 1 ), $h=(\epsilon, r)$, of length $r(r<\epsilon)$. Suppose that the arcs $T_{h, j}$ are of the same material specified by the coefficient $k_{1}$ and the union of $Y_{\epsilon, j} \backslash T_{h, j}$ (band 2) is of another material specified by the coefficient $k_{2}$ (see (13)). Let us denote $T_{h}=\cup T_{h, j}$ and $(\partial \Omega)_{h}=\cup\left(Y_{\epsilon, j} \backslash T_{h, j}\right)$.

The height of the liquid is stabilizing when it satisfies the minimization problem (see $[\mathbf{3}],[\mathbf{4}])$ :

$$
\operatorname{Min}\left\{F_{h}(v), v \in B V(\Omega)\right\},
$$

where

$$
F_{h}(v)=\int_{\Omega_{h}} \sqrt{1+|D v|^{2}}+c \int_{\Omega} v^{2}-k_{1} \int_{T_{h}} v-k_{2} \int_{(\partial \Omega)_{h}} v
$$

for $v \in B V(\Omega) ; c$ is the capillary constant, $k_{1}, k_{2}$ are respectively the cosine of the contact angles (liquid, band 1) and (liquid, band 2). $c, k_{1}$, $k_{2}$ are supposed to be positive constants. 


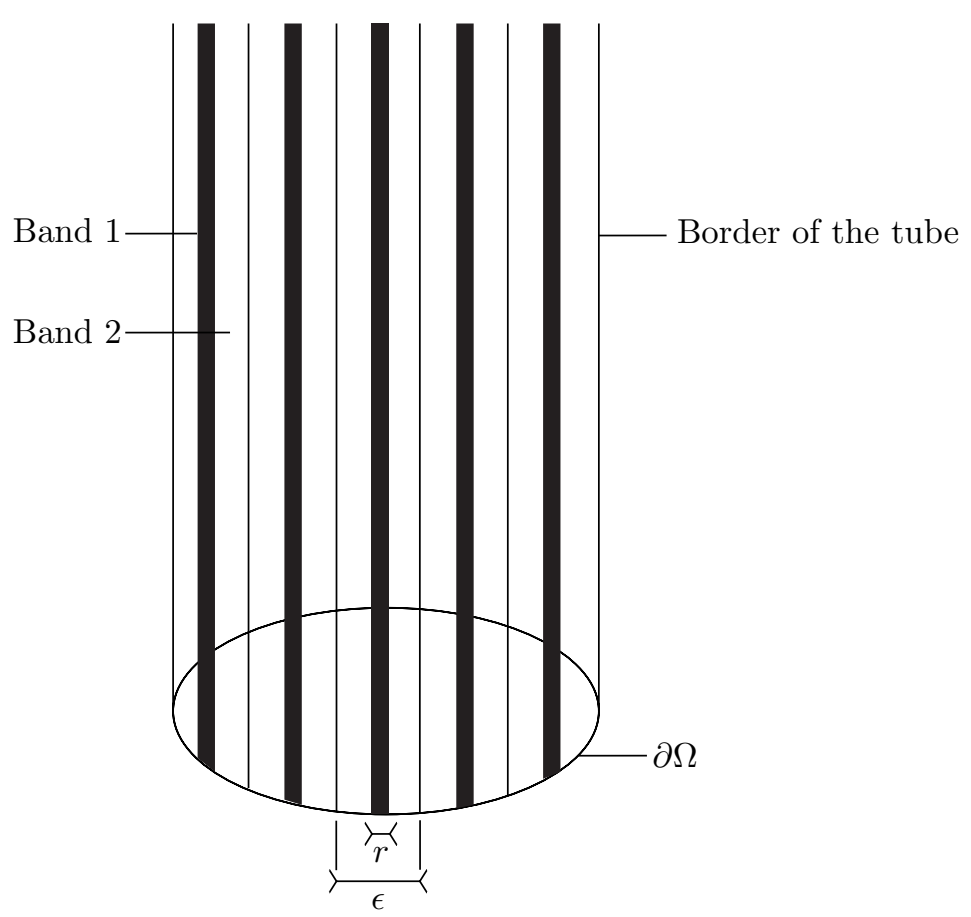

Figure 3. The tube with a strained border.

\subsection{Existence.}

Proposition 5. The problem (13) has a unique positive solution in $B V(\Omega), u_{h} \in C^{2}\left(\overline{\Omega_{h}}\right)$ and $u_{h}$ is bounded in $B V(\Omega)$. In particular $a$ subsequence, noted $u_{h}$ converges to an element $u(u \in B V(\Omega))$ in $L^{1}(\Omega)$.

Proof: The proof of the existence is similar than the one of Proposition 1.

The problem (13) is equivalent to the equation

$$
\begin{aligned}
& -\operatorname{Div} T u_{h}+2 c u_{h}=0 \quad \text { in } \quad \Omega \\
& T_{u_{h} . n}=k_{1} \quad \text { on } \quad T_{h} \\
& T u_{h} . n=k_{2} \quad \text { on } \quad \partial \Omega \backslash T_{h},
\end{aligned}
$$

where $T v=\frac{D v}{\sqrt{1+|D v|^{2}}}$ and $n$ the outer normal. 
Multiplying (14) by the characteristic function $\chi_{\Omega_{h}}$ and using the Green formula, (15) and (16)

$$
\int_{\Omega} u_{h}=\frac{1}{2 c}\left(k_{1}\left|T_{h}\right|+k_{2}\left|\partial \Omega \backslash T_{h}\right|\right)=\frac{|\Omega|}{2 c}\left(k_{1} \frac{r}{\epsilon}+k_{2} \frac{\epsilon-r}{\epsilon}\right) .
$$

The inequality

$$
J_{h}\left(u_{h}\right) \leq J_{h}(0)=|\Omega|
$$

and (see Lemma 1)

$$
\int_{\partial \Omega} u_{h} \leq \int_{\Omega}\left|D u_{h}\right|+\frac{c t}{R} \int_{\Omega} u_{h}
$$

yield

$$
\left(1-k_{3}\right) \int_{\Omega}\left|D u_{h}\right|+c \int_{\Omega} u_{h}^{2} \leq \frac{c t . k_{3}}{R} \int_{\Omega} u_{h}+|\Omega|
$$

where $k_{3}=\max \left(k_{1}, k_{2}\right)$. The boundedness of $u_{h}$ in $B V(\Omega)$ is then a consequence of (17) and (18).

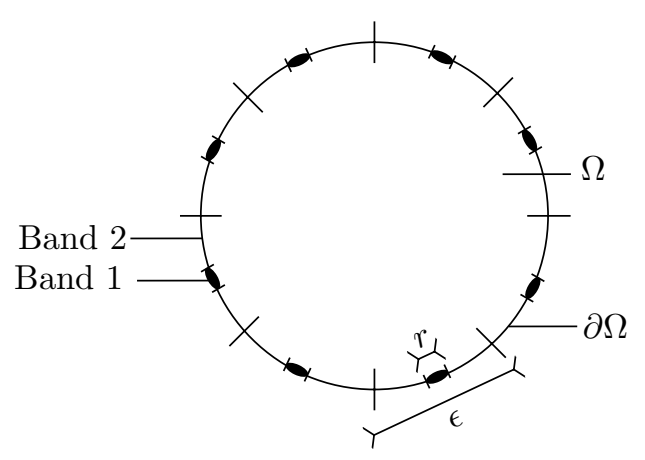

Figure 4

\subsection{Epi-limit of the functional $F_{h}$.}

The following proposition characterizes the epi-limit of the sequence $F_{h}$ when $h \rightarrow(0,0)$ :

Proposition 6. The sequence of the functionals $F_{h}$ epi-converges to the functional $F$ in $B V(\Omega)$ endowed within the topology of $L^{1}(\Omega)$ where

$$
F(v)=\int_{\Omega} \sqrt{1+|D v|^{2}}+c \int_{\Omega} v^{2}-\left[k_{1} a+k_{2}(1-a)\right] \int_{\partial \Omega} v
$$

for $v \in B V(\Omega) ; a=\lim _{h \rightarrow(0,0)} \frac{r}{\epsilon} \in[0,1]$. 
Proof: 1) Let $v \in B V(\Omega)$ and $v_{h}=v$. Since

$$
\left.\int_{T_{h}} v=\frac{r}{\epsilon} \sum_{j \in I} \epsilon\left(\frac{1}{\left|T_{h, j}\right|}\right) \int_{\partial T_{h, j}} v\right)
$$

and

$$
\left.\int_{\partial \Omega \backslash T_{h}} v=\frac{\epsilon-r}{\epsilon} \sum_{j \in I} \epsilon\left(\frac{1}{\left|Y_{j} \backslash T_{h, j}\right|}\right) \int_{Y_{j} \backslash T_{h, j}} v\right)
$$

then

$$
\lim _{h \rightarrow 0} F_{h}\left(v_{h}\right)=F(v)
$$

2) Let $v$ and $v_{h} \in B V(\Omega)$ such that $v_{h}$ converges to $v$ in $L^{1}(\Omega)$ when $h$ tends to $(0,0)$.

Using Lemma 1

$$
\begin{aligned}
F_{h}(v)-F_{h}\left(v_{h}\right) \leq & \left(\int_{\Omega} \sqrt{1+|D v|^{2}}-\int_{\Omega} \sqrt{1+\left|D v_{h}\right|^{2}}\right) \\
& +\int_{\partial \Omega}\left|v_{h}-v\right|+c\left(\int_{\Omega} v^{2}-\int_{\Omega_{h}} v_{h}^{2}\right) \\
\leq & \left(\int_{\Omega} \sqrt{1+|D v|^{2}}-\int_{\Omega} \sqrt{1+\left|D v_{h}\right|^{2}}\right) \\
& +c t \frac{1}{R} \int_{\Omega}\left|v_{h}-v\right|+c\left(\int_{\Omega} v^{2}-\int_{\Omega} v_{h}^{2}\right) .
\end{aligned}
$$

Now, passing to the limitinf in (19), using the lower semi continuity of the functional $v \rightarrow \int_{\Omega} \sqrt{1+|D v|^{2}}$ in $B V(\Omega)$ according to the topology of $L^{1}(\Omega)$ (see $[\mathbf{5}]$ ), Fatou Lemma and the fact that $v_{h}$ converges to $v$ in $L^{1}(\Omega)$, one deduces that

$$
F(v)=\liminf _{h \rightarrow 0} F_{h}(v) \leq \liminf _{h \rightarrow 0} F_{h}\left(v_{h}\right) .
$$

\subsection{Limit behavior.}

From Proposition 6, one deduces the limit behavior of the problem (13).

Theorem 2. i) The minimum of $F_{h}$ converges, when $h$ tends to $(0,0)$, to the minimum of $F$ in $B V(\Omega)$ where

$$
F(v) \int_{\Omega} \sqrt{1+|D v|^{2}}+c \int_{\Omega} v^{2}-\left[k_{1} a+k_{2}(1-a)\right] \int_{\partial \Omega} v
$$

for $v \in B V(\Omega)$ and $a=\lim _{h \rightarrow 0} \frac{r}{\epsilon} \in[0,1]$.

ii) The functional $F$ attains its minimum at the point $u$ and $u_{h}$ converges to $u$ in $L^{1}(\Omega)$ when $h$ tends to $(0,0)$. 
Physic interpretation. When the thickness $r$ of the band 1 satisfies $r \ll \epsilon(a=0)$, the tube behaves like an homogeneous one whose border is made only of the band 2 ; when $r \simeq \epsilon$, it behaves like an homogeneous one whose border is made only of the band 1 , and when $r \simeq a \epsilon, a \in(0,1)$, it behaves like an intermediate homogeneous one made only of a third material specified by its contact angle (liquid, border) whose cosine is $k_{1} a+k_{2}(1-a)$.

\section{References}

1. H. Аттоuch, "Variational convergence for functions and operators," Applicable Mathematics Series, Pitman, 1984.

2. E. De Georgi, Convergence problems for functions and operators, in "Recent methods in nonlinear analysis," Rene 1978, ed. E. De Georgi, Magenes, Mosco, Pitagora, Bologna, 1979, pp. 131-188.

3. M. Emmer, Esistenza unicita' e regolarita' nelli superfici di equilibrio nei capillari, Ann. Univ. Ferrara Sez. VII (N.S.) 18 (1973), 79-94.

4. C. Gerhardt, Existence and regularity of capillary problem, Boll. Un. Mat. Ital. (4) 10 (1974), 317-335.

5. E. Giusti, "Minimal surfaces and functions of bounded variation," Notes on Pure Mathematics 10, Australian Nat. Univ. Canberra, 1977.

6. E. Giusti, Generalised solutions for the mean curvature equation, Pacific J. Math. 88(2) (1980), 297-321.

\author{
N. Labani: \\ Department of Mathematics \\ Faculty of Sciences \\ B.P. 20 \\ Eljadida \\ MAROC
}

\author{
M. Mabrouk: \\ Laboratoire de Mécanique Appliquée \\ U.F.R. des Sciences et des Techniques \\ Route de Gray \\ 25030 Besançon Cedex \\ FRANCE
}

Primera versió rebuda el 10 d'abril de 1997, darrera versió rebuda el 27 d'abril de 1998 